\title{
Exact BER performance of asynchronous MC-DS-CDMA over Nakagami-m fading channels
}

\author{
Besma Smida ${ }^{1}$, Lajos Hanzo ${ }^{2}$, and Sofiène Affes $^{3}$ \\ 1 School of Engineering and Applied Sciences, Harvard University, Cambridge, USA \\ 2 School of Electronics and Computer Science, University of Southampton, UK \\ 3 INRS-EMT, University of Quebec, Montreal, Canada
}

\begin{abstract}
In this contribution an accurate average bit error rate (BER) formula is derived for Nakagami-faded MC-DSCDMA in the context of asynchronous transmissions and random spreading sequences. Our analysis is based on the Characteristic Function (CF) and does not rely on any assumption concerning the statistical behavior of the interference. We develop a new closed-form expression for the conditional $\mathrm{CF}$ of the inter-carrier interference and provide a procedure for calculating the exact BER expressed in the form of a single numerical integration. The accuracy of the standard Gaussian approximation (SGA) technique is also evaluated. Link-level results confirm the accuracy of the SGA for most practical conditions.
\end{abstract}

\section{INTRODUCTION}

Multi-Carrier Direct-Sequence Code Division Multiple Access (MC-DS-CDMA) [1], [2] constitutes a particularly attractive design alternative for next-generation wireless communications, since it has numerous reconfigurable parameters, which may be adjusted for the sake of satisfying diverse design goals. The Bit-Error Rate (BER) is considered to be one of the most important performance measures for communication systems and hence it has been extensively studied. The multiple access interference (MAI) and the inter-symbol interference (ISI) also affect the attainable performance of MC-DS-CDMA systems. Additionally, the MC-DS-CDMA system's performance is also affected by the inter-carrier interference (ICI). When analyzing the BER performance of MC-DS-CDMA systems, the interference sources, namely, the MAI, the ISI and the ICI are commonly assumed to be Gaussian distributed [1], [2], [3], [4]. However, the accuracy of the Gaussian approximation technique depends on the specific configuration of the system. It is widely recognized that the Gaussian approximation techniques become less accurate when a low number of users is supported or when there is a dominant interferer, creating a near-far-scenario [5].

Therefore, the employment of an accurate BER analysis dispensing with the previous assumptions concerning the distribution of the interfering sources is desirable. In order to avoid the limited accuracy of the Gaussian approximation of the interference, the BER can be calculated in the transform domain. More specifically, two widely used transforms of the decision variable's Probability Density Function (PDF) are its Fourier and Laplace transforms, corresponding to the characteristic function (CF) and the moment generating function (MGF), respectively. The basic philosophy of exact BER calculation is that first the CF or MGF of the decision variable is derived, then the associated inverse transform is performed for calculating the BER, as detailed for example in [6]. Since the decision variable can be exactly described by its CF or MGF, the BER can be accurately evaluated using numerical integration techniques. For this reason, CFand MGF-based methods have received considerable attention. The CF method was employed to study the BER performance of DS-CDMA using random sequences in both Rayleigh [6] and Nakagami-fading [7],[8] channels. The CF-method was also used to study the BER performance of MC-DS-CDMA with random sequence in Rayleigh channel [12]. A saddle point integration-based MGF oriented approach was proposed for computing the error probability of DS-CDMA systems communicating over both Rician [9] and Nakagami-fading [10] channels. This approach has been applied for studying the performance of MC-DS-CDMA systems using deterministic spreading sequences [11].

However, to the best of the authors' knowledge, the accurate BER analysis of asynchronous Nakagami-faded MCDS-CDMA using random sequences is still an open problem. Hence in this paper, we will derive an accurate BER formula for Nakagami-faded MC-DS-CDMA in the context of asynchronous transmissions and random spreading sequences. Our analysis is based on the CF, and does not rely on any simplifying assumptions concerning the statistical behavior of the interference. A new closed-form expression, rather than an integral [11] is derived for the conditional CF of the intercarrier interference (ICI).

The outline of the paper is as follows. In Section II our system and channel models are introduced, followed by the accurate analysis of Section III. The classic Gaussian approximation and our performance comparisons are provided in Section IV and Section V respectively. We conclude in Section VI.

\section{System AND Channel Model}

\section{A. MC-DS-CDMA Transmitter}

We consider an asynchronous MC-DS-CDMA system using BPSK modulation, random spreading sequences and rectangular chip waveforms. The input information sequence of the $k$-th user is first converted into $U$ parallel data sequences $b_{u}^{k}(t)$ for $u=1,2, \ldots, U$. The data sequence $b_{u}^{k}(t)=$ $\sum_{i=-\infty}^{\infty} b_{u, i}^{k} P_{T_{s}}\left(t-i T_{s}\right)$ consists of a sequence of mutually independent rectangular pulses of duration $T_{s}$ and of amplitude +1 or -1 with equal probability. After serial-to-parallel 
This full text paper was peer reviewed at the direction of IEEE Communications Society subject matter experts for publication in the WCNC 2008 proceedings.

conversion, the $u$-th substream BPSK-modulates a subcarrier frequency $f_{u}$. Then, the $U$ modulated subcarriers are superimposed, in order to form the complex-valued modulated signal. Finally, spectral spreading is imposed on the complex signal by multiplying it with a spreading code. Therefore, the transmitted signal of the $k$-th user is given by:

$$
s^{k}(t)=\sum_{u=1}^{U} \sqrt{2 P} b_{u}^{k}(t) a^{k}(t) \cos \left(2 \pi f_{u} t+\phi_{u}^{k}\right),
$$

where $P$ represents the transmitted power per subcarrier, while $a^{k}(t)$ and $\phi_{u}^{k}$ represent the spreading-code segment and the phase angle introduced in the carrier modulation process. The spreading sequence can be expressed as $a^{k}(t)=$ $\sum_{l=-\infty}^{\infty} a_{l}^{k} P_{T_{c}}\left(t-l T_{c}\right)$, where $a_{l}^{k}$ assumes values of +1 or -1 with equal probability, while $P_{T_{c}}(t)$ is the rectangular chip waveform that is defined over the interval $\left[0, T_{c}\right)$, where $T_{c}=\frac{T_{s}}{L}$ is the chip duration, while $L$ is the spreading factor.

For MC-DS-CDMA, the modulated subcarriers are orthogonal over the chip duration. Hence, the frequency corresponding to the $u$-th subcarrier is $f_{u}=f_{p}+u / T_{c}$, where $f_{p}$ is the fundamental carrier frequency. Hence, the minimum spacing between two adjacent subcarriers equals $1 / T_{c}$, which is a widely used assumption and is the case considered in this paper. If we assume that no subcarrier overlap is present, then the minimum spacing between two adjacent subcarriers should be $2 / T_{c}$.

\section{B. Channel Model}

We assume that the channel between the $k$-th transmitter and the corresponding receiver is a slowly flat Nakagami-m fading channel for each subcarrier. The channel impulse response for the $k$-th transmitted signal over the $u$-th subcarrier is given by

$$
H_{u}^{k}(t)=h_{u}^{k} \delta\left(t-\tau_{k}\right) \exp \left(-j \psi_{u}^{k}\right),
$$

where $h_{u}^{k}, \tau_{k}$ and $\psi_{u}^{k}$ represent the attenuation factor, delay and phase-shift, respectively. The delay $\tau_{k}$ of the $k$-th user is assumed to be uniformly distributed over $\left[0, T_{s}\right)$. The Nakagami-m distribution is a versatile statistical distribution, which is capable of modeling a variety of fading environments, such as land mobile, as well as indoor mobile multipath propagation channels and ionospheric radio links [13]. The PDF of the Nakagami-m fading amplitude is given by:

$$
f_{h}(x)=\frac{2}{\Gamma(m)}\left(\frac{m}{\Omega}\right)^{m} x^{2 m-1} \exp \left(-\frac{m}{\Omega} x^{2}\right), \quad x \geq 0,
$$

where $\Omega=2 \sigma^{2}$. The Nakagami-m fading family includes channels as diverse as the one-sided Gaussian fading associated with $m=1 / 2$, the Rayleigh fading having $m=1$, and no fading, corresponding to $m=\infty$. The distribution of the Nakagami-m fading phase is assumed to be uniform over $[0,2 \pi)$.

We consider $K$ asynchronous MC-DS-CDMA users, all of whom have the same number of subcarriers $U$ and the same spreading factor $L$. The average power received from each user is also assumed to be the same. Consequently, the received signal may be written as:

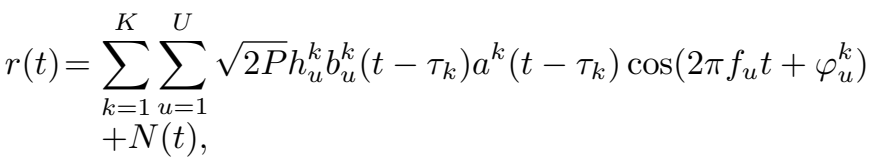

where $\varphi_{u}^{k}=\phi_{u}^{k}-\psi_{u}^{k}-2 \pi f_{u} \tau_{k}$, which is assumed to be an i.i.d. random variable having a uniform distribution over $[0,2 \pi)$, while $N(t)$ represents the additive white Gaussian noise (AWGN) with zero mean and double-sided power spectral density of $N_{o} / 2$.

\section{ACCURATE PERformance AnAlysis}

\section{A. Decision variable statistics}

Consider using a conventional single-user matched filter for coherently demodulating the desired user's signal. We assume, without loss of generality, that the reference user's index is $k=1$ and that we have $\tau_{1}=0, P=\Omega=2$ and $T_{c}=1$. The decision variable of the first user over the $v$-th subcarrier is:

$$
\begin{aligned}
Z_{v} & =\int_{0}^{T_{s}} r(t) \times a^{1}(t) \cos \left(2 \pi f_{v} t+\varphi_{v}^{1}\right) d t \\
& =D_{v}+\sum_{k=2}^{K} I_{1}^{k}+\sum_{k=2}^{K} \sum_{u=1, u \neq v}^{U} I_{2}^{k}+N_{v},
\end{aligned}
$$

where $D_{v}=h_{v}^{1} b_{v, 0}^{1} L$ is the desired output, $I_{1}^{k}$ is the interference imposed by user $k$ activating the same carrier $v, I_{2}^{k}$ is the inter-carrier interference imposed by the adjacent carriers $(u \neq v)$ of user $k$, and $N_{v}$ is the noise, which is a zero-mean Gaussian random variable with a variance $\sigma_{n}^{2}=N_{o} L / 4$.

1) Same Subcarrier Interference: The same carrier interference $\sum_{k=2}^{K} I_{1}^{k}$, where $I_{1}^{k}=h_{v}^{k} \cos \left(\theta_{v}^{k}\right) W^{k}$ and $\theta_{v}^{k}=\varphi_{v}^{1}-\varphi_{v}^{k}$, are identical to the interference that affects DS-CDMA. In their impressive study of random spreading sequences used for DSCDMA, Lehnert and Pursley [14] simplified the expression of the random variable $W^{k}$. For a rectangular chip waveform, the random variable $W^{k}$ was further simplified by Cheng and Beaulieu in [6] as follows:

$$
W^{k}=P^{k} \nu_{k}+Q^{k}\left(1-\nu_{k}\right)+X^{k}+Y^{k}\left(1-2 \nu_{k}\right),
$$

where $\nu_{k}$ is a random variable (RV) uniformly distributed over $[0,1)$, while $P^{k}$ and $Q^{k}$ are symmetric Bernoulli RVs. Furthermore, $X^{k}$ is a discrete RV that represents the sum of $A$ independent symmetric Bernoulli RVs and $Y^{k}$ is a discrete RV that represents the sum of $B$ independent symmetric Bernoulli RVs. Note that $A+B=L-1$ and the marginal PDFs of $X^{k}$ and $Y^{k}$ are given by:

$$
\begin{aligned}
P_{X^{k}}(j) & =\left(\begin{array}{c}
A \\
\frac{j+A}{2}
\end{array}\right) 2^{-A}, \\
j \in \mathcal{A} & =\{-A,-A+2, \ldots, A-2, A\}
\end{aligned}
$$

and

$$
\begin{aligned}
P_{Y^{k}}(j) & =\left(\begin{array}{c}
B \\
\frac{j+B}{2}
\end{array}\right) 2^{-B}, \\
j \in \mathcal{B} & =\{-B,-B+2, \ldots, B-2, B\} .
\end{aligned}
$$


2) Other Subcarrier Interference: The aim of this section is to simplify the expression of the inter-carrier interference. This interference is given by $\sum_{k=2}^{K} \sum_{u=1, u \neq v}^{U} I_{2}^{k}$, where $I_{2}^{k}=$ $h_{u}^{k} W_{u-v}^{k}$. We derive the random variable $W_{u-v}^{k}$ as follows:

$$
W_{u-v}^{k}=b_{u,-1}^{k} R_{k, 1, u-v}\left(\tau_{k}, \theta_{u}^{k}\right)+b_{u, 0}^{k} \hat{R}_{k, 1, u-v}\left(\tau_{k}, \theta_{u}^{k}\right),
$$

where $\theta_{u}^{k}=\varphi_{v}^{1}-\varphi_{u}^{k}$ and $R_{k, 1, u-v}\left(\tau_{k}, \theta_{u}^{k}\right)$ and $\hat{R}_{k, 1, u-v}\left(\tau_{k}, \theta_{u}^{k}\right)$ are the partial cross-correlation functions defined by:

$$
\begin{aligned}
R_{k, 1, u-v}\left(\tau_{k}, \theta_{u}^{k}\right)= & \int_{0}^{\tau_{k}} a^{k}\left(t-\tau_{k}\right) a^{1}(t) \\
& \cdot \cos \left(2 \pi\left(f_{u}-f_{v}\right) t+\theta_{u}^{k}\right) d t, \\
\hat{R}_{k, 1, u-v}\left(\tau_{k}, \theta_{u}^{k}\right)= & \int_{\tau_{k}}^{T_{s}} a^{k}\left(t-\tau_{k}\right) a^{1}(t) \\
& \cdot \cos \left(2 \pi\left(f_{u}-f_{v}\right) t+\theta_{u}^{k}\right) d t .
\end{aligned}
$$

We followed the same methodology as that used in [14] and invoked Equation (23) of [1] to simplify $W_{u-v}^{k}$ as

$$
\begin{aligned}
W_{u-v}^{k} & =\cos \left(\theta_{u}^{k}+\pi \nu_{k}|u-v|\right) \\
\cdot & {\left[\left(P^{k}-Q^{k}\right) \nu_{k} \operatorname{sinc}\left(\nu_{k}|u-v|\right)-2 Y^{k} \nu_{k} \operatorname{sinc}\left(\nu_{k}|u-v|\right)\right] } \\
& =\cos \left(\theta_{u}^{k}+\pi \nu_{k}|u-v|\right) \mathcal{W}_{u-v}^{k},
\end{aligned}
$$

where $\left(\theta_{u}^{k}+\pi \nu_{k}|u-v|\right)$ is a RV uniformly distributed over $[0,2 \pi)$. Note that $X^{k}$ does not feature in the formulation of $W_{u-v}^{k}$, which will reduce the complexity of the BER calculation.

\section{B. Same Subcarrier Interference Analysis}

The next task is the determination of the conditional $\mathrm{CF}$ of the interference $I_{1}^{k}$ incurred by user $k$ activating the same subcarrier. The $\mathrm{CF} \Phi_{I_{1}^{k} \mid B}^{N}$ of the interference $I_{1}^{k}$ encountered for transmission over a Nakagami-m fading channel has been studied in [8] for integer values of $m$ and in [15] for arbitrary values of $m$. We use Eqs. (17)-(18) of [15] to evaluate $\Phi_{I_{1}^{k} \mid B}^{N}$ as follows:

$$
\begin{aligned}
\Phi_{I_{1}^{k} \mid B}^{N}(w)= & \frac{2^{-(N-1)}}{4} \sum_{i \in \mathcal{A}} \sum_{j \in \mathcal{B}}\left(\begin{array}{c}
A \\
\frac{i+A}{2}
\end{array}\right)\left(\begin{array}{c}
B \\
\frac{j+B}{2}
\end{array}\right) \\
\times & {\left[J_{1}^{N}(i+1, j)+J_{1}^{N}(i, j-1)\right.} \\
& \left.+J_{1}^{N}(i, j+1)+J_{1}^{N}(i-1, j)\right]
\end{aligned}
$$

where

$$
\begin{aligned}
& J_{1}^{N}(i, j)= \\
& \left\{\begin{array}{lc}
{ }_{1} \mathbb{F}_{1}\left(m, 1,-\frac{i^{2}}{2 m} w^{2}\right), & j=0 \\
\frac{1}{2 j|w|}\left\{(i-j)_{2} \mathbb{F}_{2}\left(m, \frac{1}{2} ; 1, \frac{3}{2},-\frac{1}{2 m} w^{2}(i-j)^{2}\right)\right. \\
\left.-(i+j)_{2} \mathbb{F}_{2}\left(m, \frac{1}{2} ; 1, \frac{3}{2},-\frac{1}{2 m} w^{2}(i+j)^{2}\right)\right\}
\end{array},(j, w \neq 0)\right.
\end{aligned},
$$

and ${ }_{p} \mathbb{F}_{q}$ is the generalized hypergeometric function [16].

\section{Other Subcarrier Interference Analysis}

The only task that remains unsolved is the determination of the conditional $\mathrm{CF}$ of the interference $I_{2}^{k}$ incurred by user $k$ activating the other subcarriers. We have $I_{2}^{k}=h_{u}^{k} \cos \left(\theta_{u}^{k}+\right.$ $\left.\pi \nu_{k}|u-v|\right) \mathcal{W}_{u-v}^{k}$, where $h_{u}^{k}$ follows the Nakagami-m distribution, $\left(\theta_{u}^{k}+\pi \nu_{k}|u-v|\right)$ is the random phase uniformly distributed over $[0,2 \pi)$ and $\mathcal{W}_{u-v}^{k}$ is defined in Eq. (15). Upon exploiting the results of appendix A in [17], we have the CF of the ICI $I_{2}^{k}$ given $\nu_{k}$ and $B$ in the following form:

$$
\begin{aligned}
\Phi_{I_{2}^{k} \mid \nu_{k}, B}^{N}(w) & =\frac{2^{-B}}{4} \sum_{j \in \mathcal{B}}\left(\begin{array}{c}
B \\
\frac{J+B}{2}
\end{array}\right) \\
& \sum_{l=1,2,3,4}{ }_{1} \mathbb{F}_{1}\left(m, 1,-\frac{1}{2 m} \sigma_{l}^{2}\left(j, \nu_{k}, u-v\right) w^{2}\right),
\end{aligned}
$$

where

$$
\begin{aligned}
\sigma_{1}^{2}\left(j, \nu_{k}, u-v\right) & =\left[2 j \nu_{k} \operatorname{sinc}\left(\nu_{k}|u-v|\right)\right]^{2}, \\
\sigma_{2}^{2}\left(j, \nu_{k}, u-v\right) & =\left[2 j \nu_{k} \operatorname{sinc}\left(\nu_{k}|u-v|\right)\right]^{2}, \\
\sigma_{3}^{2}\left(j, \nu_{k}, u-v\right) & =\left[2(1-j) \nu_{k} \operatorname{sinc}\left(\nu_{k}|u-v|\right)\right]^{2}, \\
\sigma_{4}^{2}\left(j, \nu_{k}, u-v\right) & =\left[2(1+j) \nu_{k} \operatorname{sinc}\left(\nu_{k}|u-v|\right)\right]^{2} .
\end{aligned}
$$

The variables $\nu_{k}$ now appear in the numerator of the function ${ }_{1} \mathbb{F}_{1}$ and averaging can be carried out by using Eq. (7.512) of [16], yielding the CF of $I_{2}^{k}$ given $B$ as:

$$
\begin{aligned}
\Phi_{I_{2}^{k} \mid B}^{N}(w)= & \int_{0}^{1} \Phi_{I_{2}^{k} \mid \nu_{k}, B}^{N}(w) d \nu_{k} \\
= & \frac{2^{-B}}{4} \sum_{j \in \mathcal{B}}\left(\begin{array}{c}
B \\
\frac{j+B}{2}
\end{array}\right) \\
& \times\left[2 J_{2}^{N}(j)+J_{2}^{N}(j-1)+J_{2}^{N}(j+1)\right]
\end{aligned}
$$

where $B$ varies from 0 to $L-1$, and

$J_{2}^{N}(j)=\frac{1}{\pi} \Gamma(0.5) \Gamma(0.5){ }_{2} \mathbb{F}_{2}\left(\frac{1}{2}, m ; 1,1 ;-\frac{1}{2 m} \frac{4 j^{2} w^{2}}{\pi^{2}(u-v)^{2}}\right)$.

The detailed derivation of the above expressions is provided in the appendix.

\section{Exact BER Analysis}

After evaluating the $\mathrm{CF}$ of the total interference, we can derive the average BER under asynchronous MC-DS-CDMA transmission conditions. Indeed, we will apply the generalized equation (4) of [15] to calculate the BER for Nakagami-m fading. The $\mathrm{CF}$ of the total interference over a Nakagami-m fading channel is given by:

$$
\Phi_{I \mid B}^{N}(w)=\Pi_{k=2}^{K}\left[\Phi_{I_{1}^{k} \mid B}^{N}(w) \Pi_{u=1, u \neq v}^{U} \Phi_{I_{2}^{k} \mid B}^{N}(w)\right] .
$$

The conditional BER evaluated for transmission over a Nakagami-m fading channel can be expressed as [17] (assuming $m$ takes only integer values):

$$
\begin{aligned}
P_{e \mid B}= & \frac{1}{2^{m}}\left[1-\frac{L}{\sqrt{L^{2}+m \sigma_{n}^{2}}}\right]^{m} \sum_{k=0}^{m-1} 2^{-k}\left(\begin{array}{c}
m-1+k \\
k
\end{array}\right) \\
& \cdot\left[1+\frac{L}{\sqrt{L^{2}+m \sigma_{n}^{2}}}\right]^{k}+\frac{L}{\pi} \frac{\Gamma\left(m+\frac{1}{2}\right)}{\Gamma(m)} \sqrt{\frac{2}{m}} \\
& \cdot \int_{0}^{+\infty}\left[1-\Phi_{I \mid B}^{N}(w)\right]_{1} \mathbb{F}_{1}\left(m+\frac{1}{2}, \frac{3}{2},-\frac{L^{2} w^{2}}{2 m}\right) d w .
\end{aligned}
$$


Finally, the overall average BER is obtained by averaging $P_{e \mid B}$ over all spreading sequences:

$$
P_{e}=2^{-(L-1)} \sum_{B=0}^{L-1}\left(\begin{array}{c}
L-1 \\
B
\end{array}\right) P_{e \mid B} .
$$

\section{STANDARD GAUSSIAN APPROXIMATION}

Using the derivations found in [3], the average BER $P_{e}$ over Nakagami-m fading approximated by the SGA can be shown to be (assuming that $m$ takes only integer values):

$$
P_{e}=\left[\frac{1-\mu}{2}\right]^{m} \sum_{k=0}^{m-1}\left(\begin{array}{c}
m-1+k \\
k
\end{array}\right)\left[\frac{1+\mu}{2}\right]^{k},
$$

where

$$
\mu=\sqrt{\gamma /(\gamma+m)}
$$

and

$$
\begin{aligned}
\gamma^{-1}= & \frac{N_{o}}{4 L}+\frac{2(K-1)}{3 L} \\
& +(K-1)\left[\frac{1}{U} \sum_{u=1}^{U} \sum_{u=1, u \neq v}^{U} \frac{1}{\pi^{2}(u-v)^{2} L}\right] .
\end{aligned}
$$

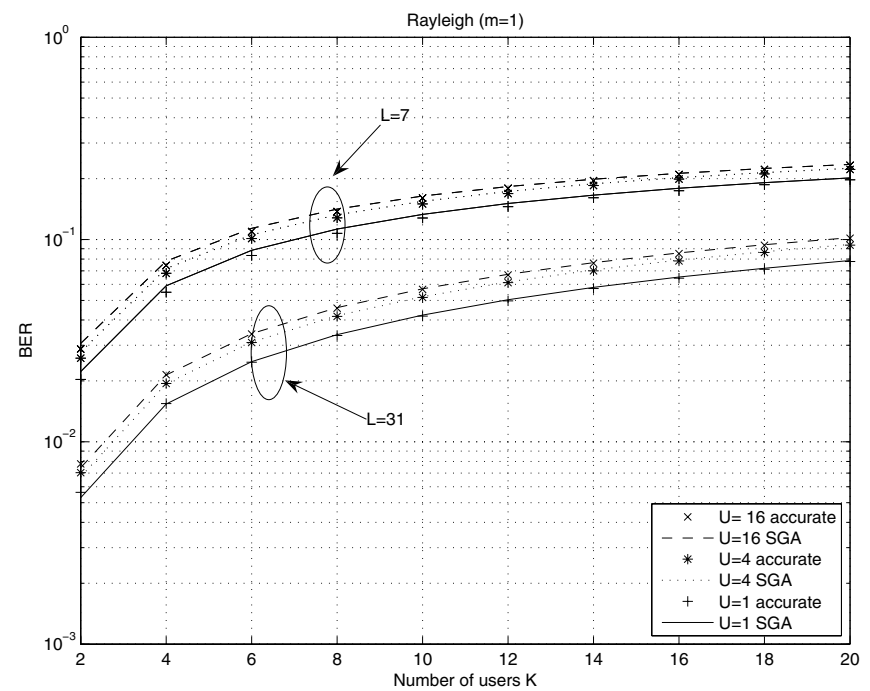

Fig. 1. BER versus the number of users $K$ in an asynchronous MC-DS CDMA system exposed to Rayleigh fading ( $m=1$ ), using random spreading sequences and BPSK modulation. The length of the spreading sequence is $L=7$ and $L=31$. The number of subcarriers is $U=1,4$, and 16 . The average power of all subcarriers and users is equal and the background noise is ignored.

\section{Numerical RESUlts}

This performance evaluation is not an approximation relying on central limit theorem arguments, but rather an arbitrarily tight calculation with an accuracy controlled by the resolution of the numerical integration in Eq. (24). Section III and the appendix give the proof of our exact BER formula. In this section we will compare the results obtained from our accurate BER analysis to those generated by the SGA. Since the evaluation of the ICI effects on the performance of MCDS-CDMA is the main objective of our analysis, we assume first that the effect of noise is negligible.

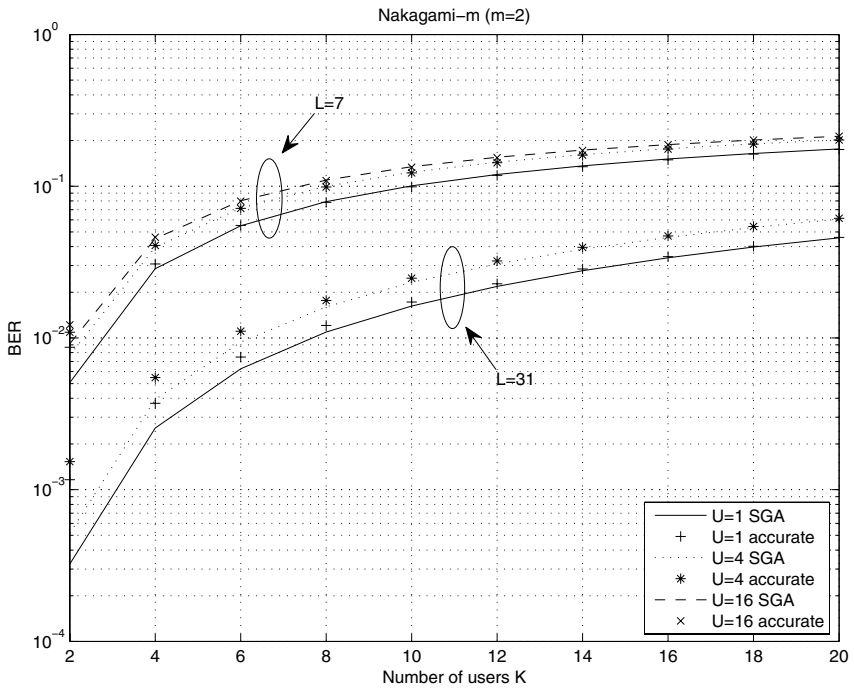

Fig. 2. BER versus the number of users $K$ in an asynchronous MC-DSCDMA system exposed to Nakagami-m fading $(m=2)$, using random spreading sequences and BPSK modulation. The length of the spreading sequence is $L=7$ and $L=31$. The number of subcarriers is $U=1,4$, and 16 . The average power of all subcarriers and users is equal and the background noise is ignored.

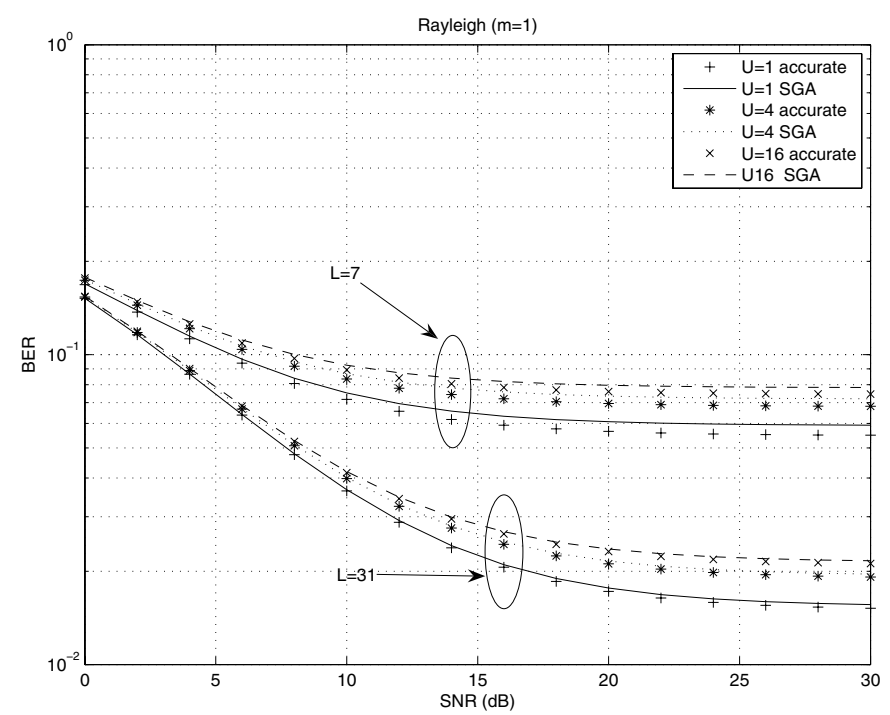

Fig. 3. BER versus the per-bit SNR in an asynchronous MC-DS-CDMA exposed to Rayleigh fading ( $m=1$ ), when using random spreading sequences and BPSK modulation. The length of the spreading sequence is $L=7$ and $L=31$. The number of subcarriers is $U=1,4$, and 9 . The average power of all subcarriers and users is equal. The number of users is $K=4$.

Figs. 1 and 2 show the average BER performance against the number of users in the context of Rayleigh $(m=1)$ and Nakagami-m $(m=2)$ fading, respectively. We observe that the SGA provides good approximation to the accurate BER computed via the characteristic function. For Rayleigh fading, the SGA slightly over-estimates the BER. By contrast, when $m=2$, the SGA under-estimates the average BER.

Comparing the results in Fig. 1, we see that the inaccuracy of the SGA becomes prevalent, when the number of users, the spreading factor, and/or the number of subcarriers decreases. 


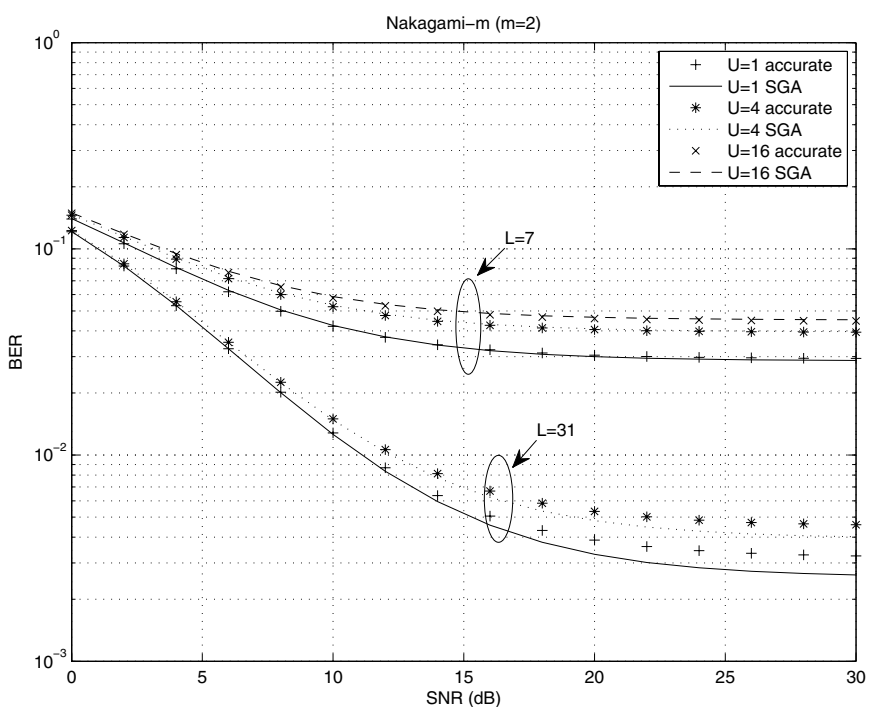

Fig. 4. BER versus the per-bit SNR in an asynchronous MC-DS-CDMA exposed to Nakagami-m fading $(m=2)$, when using random spreading sequences and BPSK modulation. The length of the spreading sequence is $L=7$ and $L=31$. The number of subcarriers is $U=1,4$, and 16 . The average power of all subcarriers and users is equal. The number of users is $K=4$.

This is different when $m=2$, Fig. 2 shows that the SGA is a better approximation for a spreading factor $L=7$ than for $L=31$.

Figs. 3 and 4 illustrate the average BER performance versus the per-bit SNR, when the number of users is $K=4$. It compares the results obtained by the SGA to our accurate BER. Figs. 3 and 4 confirm, not surprisingly, that the presence of strong background noise improves the accuracy of the SGA method.

\section{CONCLUSION}

We studied the accurate BER calculation of an asynchronous MC-DS-CDMA system using random spreading sequences and BPSK modulation. We considered a flat Nakagami-m fading channel. Using the $\mathrm{CF}$ approach, we derived a new closed-form expression for the conditional CF of the ICI and only a single integration was required for the BER calculation. For most practical scenarios the SGA remains fairly accurate, although it slightly over-estimates the average BER when the fading is severe while it under-estimates the average BER when the fading is benign.

\section{APPENDIX}

In this appendix, we derive $J_{2}^{N}(j)$ of Eq. (22). For $w \neq 0$, the integration of the first term in Eq. (18) gives

$$
\begin{aligned}
& \int_{0}^{1}{ }_{1} \mathbb{F}_{1}\left(m, 1,-\frac{1}{2 m} \sigma_{1}^{2}\left(j, \nu_{k}, u-v\right) w^{2}\right) d \nu_{k} \\
& =\int_{0}^{1}{ }_{1} \mathbb{F}_{1}\left(m, 1,-\frac{1}{2 m}\left[2 j \nu_{k} \operatorname{sinc}\left(\nu_{k}|u-v|\right)\right]^{2} w^{2}\right) d \nu_{k} \\
& =\int_{0}^{1}{ }_{1} \mathbb{F}_{1}\left(m, 1,-\frac{1}{2 m} \frac{4 j^{2} w^{2}}{\pi^{2}(u-v)^{2}} \sin ^{2}\left(\pi \nu_{k}|u-v|\right)\right) d \nu_{k}
\end{aligned}
$$

Then, we substitute the variable $\pi \nu_{k}|u-v|$ by $\theta_{k}$ and $\left[\sin \left(\theta_{k}\right)\right]^{2}$ by $y$ as

$$
\begin{aligned}
& \int_{0}^{1}{ }_{1} \mathbb{F}_{1}\left(m, 1,-\frac{1}{2 m} \frac{4 j^{2} w^{2}}{\pi^{2}(u-v)^{2}} \sin ^{2}\left(\pi \nu_{k}|u-v|\right)\right) d \nu_{k} \\
& =\frac{1}{\pi} \int_{0}^{\pi}{ }_{1} \mathbb{F}_{1}\left(m, 1,-\frac{1}{2 m} \frac{4 j^{2} w^{2}}{\pi^{2}(u-v)^{2}} \sin ^{2}\left(\theta_{k}\right)\right) d \theta_{k}, \\
& =\frac{1}{\pi} \int_{0}^{1}{ }_{1} \mathbb{F}_{1}\left(m, 1,-\frac{1}{2 m} \frac{4 j^{2} w^{2}}{\pi^{2}(u-v)^{2}} y\right) \frac{1}{\sqrt{y} \sqrt{1-y}} d y .
\end{aligned}
$$

Using Eq. (7.512) of [16], we derive the previous integration as follows:

$$
\begin{aligned}
& \frac{1}{\pi} \int_{0}^{1}{ }_{1} \mathbb{F}_{1}\left(m, 1,-\frac{1}{2 m} \frac{4 j^{2} w^{2}}{\pi^{2}(u-v)^{2}} y\right) \frac{1}{\sqrt{y} \sqrt{1-y}} d y \\
& =\frac{1}{\pi} \Gamma(0.5) \Gamma(0.5)_{2} \mathbb{F}_{2}\left(\frac{1}{2}, m ; 1,1 ;-\frac{1}{2 m} \frac{4 j^{2} w^{2}}{\pi^{2}(u-v)^{2}}\right) .
\end{aligned}
$$

\section{REFERENCES}

[1] E.A. Sourour and M. Nakagawa, "Performance of orthogonal multicarrier CDMA in a multipath fading channel," IEEE Transactions on Communications, vol. 44, no. 3, pp. 356-367, Mar. 1996.

[2] L. Hanzo, T. Keller, M. Muenster, and B.J. Choi, OFDM and MC-CDMA for Broadband Multiuser Communications, WLANs and Broadcasting, John Wiley \& Sons Inc, 2003.

[3] Y.L-L. Yang and L. Hanzo, "Performance of generalized multicarrier DS-CDMA over Nakagami-m fading channels," IEEE Transactions on Communications, vol. 50, no. 6, pp. 956-966, June 2002.

[4] S. Kondo and L.B. Milstein, "Performance of multicarrier DS CDMA systems," IEEE Transactions on Communications, vol. 44, no. 2, pp. 238-246, Feb. 1996.

[5] M.O. Sunay and P.J. McLane, " Caculating error probabilities for DSCDMA systems: when not to use the Gaussian approximation," Proc. IEEE GLOBECOM, 1996, vol. 3, pp. 1744-1749.

[6] J. Cheng and N.C. Beaulieu,"Accurate DS-CDMA bit-error probability calculation in Rayleigh fading," IEEE Transactions on Wireless Communications, vol. 1, no. 1, pp. 3-15, Jan. 2002.

[7] J. Cheng and N.C. Beaulieu, "Precise bit error rate calculation for asynchronous DS-CDMA in Nakagami fading," in Proc. IEEE GLOBECOM, 2000, vol. 2, pp. 980-984.

[8] J. Cheng and N.C. Beaulieu, "Error rate of asynchronous DS-CDMA in Nakagami fading," IEEE Transactions on Wireless Communications, vol. 4, no. 6, pp. 2674 - 2676, Nov. 2005.

[9] D. Liu, C.L. Despins and W.A. Krzymien, "Low-complexity performance evaluation of binary and quaternary DS-SSMA over Rician fading channels via the characteristic function method," Wireless Personal Commun., vol. 7, no. 2-3, pp. 257-273, Aug. 1998.

[10] S.W. Oh and K.H. Li, "Performance evaluation for forward-link cellular DS-CDMA over frequency-selective Nakagami multipath fading channels," Wireless Personal Commun., vol. 18, no. 3, pp. 275-287, Sep. 2001.

[11] B. Smida, C.L. Despins and G.Y. Delisle, "MC-CDMA performance evaluation over a multipath fading channel using the characteristic function method," IEEE Transactions on Communications, vol. 49, no. 8, pp. 1325-1328, Aug. 2001

[12] B. Smida, L. Hanzo, and S. Affes, "Accurate BER of MC-DS-CDMA over Rayleigh fading channels", Proceding of International Workshop on Multi-Carrier Spread Spectrum MC-SS'2007, pp. 147-156, May 2007.

[13] M.K. Simon, and M. Alouini, "A unified approach to the performance analysis of digital communication over generalized fading channels," Proceedings of the IEEE, vol. 86, no. 9, pp. 1860-1877, Sep. 1998.

[14] J.S. Lehnert and M.B. Pursley, "Error probabilities for binary directsequence spread spectrum communications with random signature sequences," IEEE Transactions on Communications, vol. 35, no. 1, pp. 87-98, Jan. 1987.

[15] X. Liu and L. Hanzo," A unified exact BER performance analysis of asynchronous DS-CDMA sytems using BPSK modulation over fading channels," IEEE Transactions on Wireless Communications, to appear.

[16] I.S. Gradshteyn and I.M. Ryzhik, Table of Integrals, Series, and Products, New York: Academic Press, Sixth edition, July 2000.

[17] J. Cheng and N.C. Beaulieu, "Precise error-rate analysis of bandwidthefficient BPSK in Nakagami fading and cochannel interference," IEEE Transactions on Communications, vol. 52, no. 1, pp. 149-158, Jan. 2004. 